Hui-Hsiung Kuo

Nagoya Math. J.

Vol. 50 (1973), 89-116

\title{
STOCHASTIC INTEGRALS IN ABSTRUCT WIENER SPACE II: REGULARITY PROPERTIES
}

\author{
HUI-HSIUNG KUO*
}

\section{Introduction}

This paper continues the study of stochastic integrals in abstract Wiener space previously given in [14]. We will present, among other things, the detailed discussion and proofs of the results announced in [16]. Let $H \subset B$ be an abstract Wiener space. Consider the following stochastic integral equation in $H \subset B$,

$$
X(t)=x+\int_{0}^{t} A(s, X(s)) d W(s)+\int_{0}^{t} \sigma(s, X(s)) d s,
$$

where $W(t)$ is a Wiener process in $B$. Under certain assumptions on $A$ and $\sigma$ we showed in [14] that (1) has a unique non-anticipating continuous solution and that this solution is a Markov process. If $A$ and $\sigma$ are differentiable in the second variable we can differentiate the above equation "formally" with respect to the starting point $x$ to obtain the formal operator-valued stochastic integral equation

$$
Y(t)=I+\int_{0}^{t} A_{x}(s, X(s)) Y(s) d W(s)+\int_{0}^{t} \sigma_{x}(s, X(s)) Y(s) d s,
$$

where $A_{x}$ and $\sigma_{x}$ are derivatives of $A$ and $\sigma$ in the second variable, respectively. (2) is a linear integral equation and obviously has a unique solution which qualifies to be called the derivative of $X(t)$ in some sense. If $A$ and $\sigma$ are furthermore twice differentiable we can differentiate (2) formally in the same manner to obtain another stochastic integral equation whose solution is the second derivative of $X(t)$. Thus roughly speaking, the solution $X(t)$ of (1), regarded as a function of its starting point, is as smooth as $A$ and $\sigma$.

Let $f$ be a real-valued continuous function in $B$. Let $\theta(x)=$

Received July 25, 1972.

* This work was supported in part by NSF Grant GU-3784. 
$E_{x}[f(X(t))]$. If $f$ is differentiable then formally by the "chain rule" we have $\theta^{\prime}(x)=E_{x}\left[Y(t)^{*}\left(f^{\prime}(X(t))\right)\right]$, where $Y(t)$ is the solution of (2) and * denotes the adjoint of operators of $H$. If $f$ is twice differentiable then so is $\theta$ and a formal expression for $\theta^{\prime \prime}(x)$ can be written by using also the second derivative of $X(t)$. Thus if $A$ and $\sigma$ are $C^{\infty}$-functions then $\theta$ is as smooth as $f$. Furthermore, if $f^{\prime \prime}(x)$ is a Hilbert-Schmidt operator then $\theta^{\prime \prime}(x)$ is also a Hilbert-Schmidt operator.

The above approach of discussing the regularity properties of $X(t)$ and $\theta(x)$ was first introduced by Gikhman [3;4]. It was carried over to infinite dimensional Hilbert spaces by Dalec'kii $[1 ; 2]$. See also[18;23]. We generalize it to Banach spaces (\$2) and, furthermore, study the related operator-valued stochastic integrals and prove the corresponding versions of Ito's formula and Girsanov-Skorokhod-McKean's formula (§1). In case $A$ and $\sigma$ are time-independent we show in the end of the paper that $X(t)$ generates a semi-group on the Banach space of bounded continuous functions on $B$ vanishing at infinity. The proof is due to K. Ito.

Recently, Kannan and Bharucha-Reid [10;11] have defined several operator-valued stochastic integrals and proved some generalizations of Ito's formula. However, there is no apparent relation between their work and ours.

This paper is closely related to Piech's. In a series of papers [19; $20 ; 21 ; 22]$ she studies the corresponding parabolic equation of (1) with $\sigma \equiv 0$ and $A$ satisfying stronger assumptions. In particular, $A$ is nondegenerate. She constructs a fundamental solution $\left\{q_{t}(s, d y)\right\}$ which is related to the process $X(t)$ by $\int_{B} f(y) q_{t}(x, d y)=E_{x}[f(X(t))]$ for bounded Lip-1 functions $f$ [17]. Her conclusions about the regularity properties of the function $\theta(x)=E_{x}[f(X(t))]$ are stronger than ours in this particular case.

\section{Notation}

1. $E$ expectation

2. $H \subset B \quad$ abstract Wiener space

3. $B^{*} \subset H \subset B \quad$ (through identifications)

4. $|\cdot| \quad H$-norm (see 7)

5. $\|\cdot\| \quad B$-norm (see 8)

6. $L^{n}(X ; Y) \quad$ continuous $n$-linear maps from $X \times X \times \cdots \times X$ into $Y$ 

7. $|\cdot|$
norm of $L^{n}(H ; R)$
8. $\|\cdot\|$
norm of $L^{n}(B ; R)$
9. $L_{(2)}(H ; H)$
Hilbert-Schmidt operators of $H$ (see 12)
10. $|\cdot|_{2},\langle,\rangle_{2}$
norm, inner product of $L_{(2}(H ; H)$. (see 13)
11. $\sim$
$\tilde{T}(x)=T(x, \cdot, \cdots, \cdot) . T \in L^{n}(X ; R), \tilde{T} \in L\left(X ; L^{n-1}(X ; R)\right)$
(cf. 33).

12. $L_{(2)}^{n}(H ; R)$

Hilbert-Schmidt type $n$-linear forms of $H$.

13. $|\cdot|_{2},\langle,\rangle_{2}$ norm, inner product of $L_{(2)}^{n}(H ; R)$

14. $\circ_{j}$ $S \circ_{j} T, S \in L^{n}(X ; R), T \in L(X ; X) ; S \circ_{j} T \in L^{n}(X ; R)$.

15. $\|\cdot\|_{X}$ $\left(S \circ_{j} T\left(x_{1}, \cdots, x_{j}, \cdots, x_{n}\right)=S\left(x_{1}, \cdots, T x_{j}, \cdots, x_{n}\right)\right)$

16. $W(t)$ norm of $L(X ; X)$.

17. $\mathscr{M}_{t}$ Wiener process in $B$.

18. $\mathscr{L}\left[L_{(2)}^{n}(H ; R)\right]$ non-anticipating stochastic processes $\xi$ with state space $L_{(2)}^{n}(H ; R)$ such that $\int_{0}^{\tau} E|\xi(t)|^{2} d t<\infty$ for each finite $\tau$. (see 20)

19. $\mathscr{L}\left[L^{n}(B ; R)\right]$

20. $\mathscr{L}[X]$

non-anticipating stochastic processes $\xi$ with state space $X$ such that $\int_{0}^{\tau} E|\xi(t)|_{X}^{2} d t<\infty$ for each finite $\tau$. (cf. 27)

21. $\mathscr{S}(\mathscr{H} ; \mathscr{K}) \quad$ trace-class type bilinear form from $\mathscr{H} \times \mathscr{H}$ into $\mathscr{K}$.

22. TRACE $S$ trace of $S \in \mathscr{S}(\mathscr{H} ; \mathscr{K})$.

23. $\triangle$

a) $T \in L^{n}(H ; R), S \in L\left(L^{n-1}(H ; R) ; L^{n-1}(H ; R)\right)$

$S \triangle T \in L^{n}(H ; R)$. $\left((S \Delta T)^{\sim}=S \circ \tilde{T}\right)$

c) $S \in L^{n}(H ; R), T \in L\left(L^{n-1}(H ; R) ; R\right) ; S \Delta T \in H$. $(\langle S \Delta T, h\rangle=T(\tilde{S}(h)))$.

24. $L_{1}(H ; H)$ trace class operators of $H$.

25. $X_{x}(t)$ diffusion process starting at $x$.

26. $C_{H}^{n}$

27. $\mathscr{L}(D)$ $n$-smooth functions in $H$-directions. square integrable random variables taking values in D. (cf. 20)

28. $\delta \xi_{x}$ $M S-H$-derivative of a random variable $\xi$ at $x$.

29. $M S-C_{H}^{n}$ $M S$ - $n$-smooth random variables in $H$-directions.

30. $\delta Z, \delta^{n} Z$ $M S-H$-derivative of a diffusion process $Z$. 


\begin{tabular}{|c|c|}
\hline 31. ^ & $\begin{array}{l}S \in L^{n}(H ; R), \hat{S} \in L^{n-1}(H ; H) \\
\left.\left(\left\langle\hat{S}\left(h_{1}, \cdots, h_{n-1}\right), h\right)\right\rangle=S\left(h, h_{1}, \cdots, h_{n-1}\right)\right)\end{array}$ \\
\hline 32. & $\begin{array}{l}T \in L^{3}(H ; R), S \in L^{n}(H ; R) ; S: T \in L^{n+1}(H ; R) . \\
\left(S: T\left(h_{1}, h_{2}, \cdots, h_{n}, h_{n+1}\right)=T\left(h_{1}, \hat{S}\left(h_{2}, \cdots, h_{n}\right), h_{n+1}\right)\right) .\end{array}$ \\
\hline 33. & $\begin{array}{l}S \in L^{n}(H ; R), \check{S} \in L\left(H ; L^{n-1}(H ; R)\right) \\
(\check{S}(h)=S(\cdot, \cdot, \cdots, h)) . \quad(c f .11)\end{array}$ \\
\hline
\end{tabular}

\section{Operator-Valued Stochastic Integrals}

Let $H \subset B$ be an abstract Wiener space. | | | and $\|\cdot\|$ denote the $H$ norm and $B$-norm, respectively. We will regard $B^{*} \subset H^{*} \approx H \subset B$ in the natural way. As in [14] we assume that there is a sequence $Q_{n}$ of finite dimensional projections such that (i) $Q_{n}(B) \subset B^{*}$ and (ii) $Q_{n}$ converges strongly to the identity both in $B$ and in $H$. Furthermore, we will assume that there exists an orthonormal basis $\left\{e_{n}\right\}$ of $H$ such that $\sum_{n=1}^{\infty}\left\|e_{n}\right\|^{2}<\infty$. This additional assumption is satisfied by all of the presently known abstract Wiener spaces.

\section{Notation:}

(i ) $L^{n}(X ; Y)=$ the Banach space of all continuous $n$-linear maps from $X^{n}$ into $Y$, where $X$ and $Y$ are Banach spaces. $L^{1}$ will be written as $L$. (ii) $L^{n-1}\left(X ; X^{*}\right) \approx L^{n}(X ; R)$

(iii) $\|\cdot\|$ and $|\cdot|$ denote the norms of $L^{n}(B ; R)$ and $L^{n}(H ; R)$, respectively. Clearly $L^{n}(B ; R) \subset L^{n}(H, R)$ and $|\cdot|$ is dominated by $\|\cdot\|$ with some constant depending on $n$.

(iv) $L_{(2)}(H ; H)\left(\equiv L_{(2)}^{2}(H ; R)\right)$ denotes the Hilbert space of all HilbertSchmidt operators of $H$ with $H$-S-norm $|\cdot|_{2}=\langle,\rangle_{2}^{1 / 2}$. It can be shown easily that $|S|_{2} \leq\left(\sum_{j=1}^{\infty}\left\|e_{j}\right\|^{2}\right)^{1 / 2}\|S\|$ for all $S \in L^{2}(B ; R)$, where $\left\{e_{j}\right\}$ is given in the additional assumption. Thus we have $L^{2}(B ; R) \subset L_{(2)}^{2}(H ; R)$.

(v) Let $T \in L^{n}(X ; R)$. Define $\tilde{T} \in L\left(X ; L^{n-1}(X ; R)\right)$ by $\tilde{T}(x)=T(x, \cdot, \cdot$, $\cdots, \cdot)$.

Now we want to define inductively a sequence of Hilbert spaces $L_{(2)}^{n}(H ; R), n \geq 1$, with $L_{(2)}^{1}(H ; R)=H$ by convention and $L_{(2)}^{2}(H ; R)$ given above.

Definition 1. Let $T \in L^{n}(H ; R), n \geq 3 . \quad T$ is said to be of HilbertSchmidt type if (i) $\tilde{T}(H) \subset L_{(2)}^{n-1}(H ; R)$ and (ii) $\tilde{T}$ is a Hilbert-Schmidt operator from $H$ into $L_{(2)}^{n-1}(H ; R)$. 
Let $L_{(2)}^{n}(H ; R)$ denote the space of all Hilbert-Schmidt type $n$-linear forms of $H$. It is a Hilbert space with the inner product $\langle S, T\rangle_{2}=$ the Hilbert-Schmidt inner product of $\tilde{S}$ and $\tilde{T}, S, T \in L_{(2)}^{n}(H ; R)$. Clearly,

$$
\langle S, T\rangle_{2}=\sum_{i_{1}, i_{2}, \cdots, i_{n}} S\left(v_{i_{1}}, v_{i_{2}}, \cdots, v_{i_{n}}\right) T\left(v_{i_{1}}, v_{i_{2}}, \cdots, v_{i_{n}}\right),
$$

where $\left\{v_{k}\right\}$ is any orthonormal basis of $H$. Let $|S|_{2}=\langle S, S\rangle_{2}^{1 / 2}$. Note that we have used the same notation $|\cdot|_{2}$ and $\langle,\rangle_{2}$ to denote the norm and the inner product of $L_{(2)}^{n}(H ; R)$ for all $n \geq 2$ since there is no confusion. For example, the meaning of the following equality is clear, when $S, T \in L_{(2)}^{n}(H ; R)$,

$$
\langle S, T\rangle_{2}=\sum_{k}\left\langle\tilde{S}\left(v_{k}\right), \tilde{T}\left(v_{k}\right)\right\rangle_{2} .
$$

LEMMA 1.1. (a) $|S| \leq|S|_{2}$ for all $S$ in $L_{(2)}^{n}(H ; R)$.

(b) $|T|_{2} \leq c^{n}\|T\|$ for all $T$ in $L^{n}(B ; R)$, where $c$ is a constant. Thus we have the relation $L^{n}(B ; R) \subset L_{(2)}^{n}(H ; R) \subset L^{n}(H ; R), n \geq 1$.

(c) $L^{n}(B ; R)$ is dense in $L_{(2)}^{n}(H ; R)$.

Proof. Let $\left\{v_{j}\right\}$ be an orthonormal basis of $H$. Then

$$
\begin{aligned}
S\left(h_{1}, h_{2}, \cdots, h_{n}\right)^{2} & =\left\{\sum_{j}\left(h_{1}, v_{j}\right) S\left(v_{j}, h_{2}, \cdots, h_{n}\right)\right\}^{2} \\
& \leq\left\{\sum_{j}\left(h_{1}, v_{j}\right)^{2}\right\}\left\{\sum_{j} S\left(v_{j}, h_{2}, \cdots, h_{n}\right)^{2}\right\} \\
& =\left|h_{1}\right|^{2} \sum_{j} S\left(v_{j}, h_{2}, \cdots, h_{n}\right)^{2} \\
& \leq\left|h_{1}\right|^{2}\left|h_{2}\right|^{2} \cdots\left|h_{n}\right|^{2} \sum_{i_{1}, i_{2}, \cdots, i_{n}} S\left(v_{i_{1}, i_{2}}, \cdots, v_{i_{n}}\right)^{2},
\end{aligned}
$$

whence (a) follows. To prove (b) and (c) let $\left\{Q_{n}\right\}$ and $\left\{e_{k}\right\}$ be given in the beginning of this section. Then

$$
\begin{aligned}
|T|_{2}^{2} & =\sum_{i_{1}, i_{2}, \cdots, i_{n}} T\left(e_{i_{1}}, e_{i_{2}}, \cdots, e_{i_{n}}\right)^{2} \\
& \leq \sum_{i_{1}, i_{2}, \cdots, i_{n}}\left(\|T\|\left\|e_{i_{1}}\right\|\left\|e_{i_{2}}\right\| \cdots\left\|e_{i_{n}}\right\|\right)^{2} \\
& =\left(\sum_{i}\left\|e_{i}\right\|^{2}\right)^{n}\|T\|^{2} .
\end{aligned}
$$

Moreover, if $U \in L_{(2)}^{n}(H ; R)$, let $U_{j}=U\left(Q_{j}(\cdot), Q_{j}(\cdot), \cdots, Q_{j}(\cdot)\right)$. Then $U_{j} \in L^{n}(B ; R)$ and $\left|U_{j}-U\right|_{2} \rightarrow 0$.

Example 1. Let $H=L^{2}(0,1)$ (real-valued). Suppose $\phi$ is a measurable function on $(0,1)^{n}$ such that

$$
\int_{0}^{1} \int_{0}^{1} \cdots \int_{0}^{1}\left|\phi\left(t_{1}, t_{2}, \cdots, t_{n}\right)\right|^{2} d t_{1} d t_{2} \cdots d t_{n}<\infty .
$$

Define $K: H^{n} \rightarrow R$ by 


$$
K\left(f_{1}, f_{2}, \cdots f_{n}\right)=\int_{0}^{1} \int_{0}^{1} \cdots \int_{0}^{1} \phi\left(t_{1}, t_{2}, \cdots, t_{n}\right) f_{1}\left(t_{1}\right) f_{2}\left(t_{2}\right) \cdots f_{n}\left(t_{n}\right) d t_{1} d t_{2} \cdots d t_{n} .
$$

Then $K$ is a Hilbert-Schmidt type $n$-form on $H$ and $|K|_{2}=\left[\int_{0}^{1} \int_{0}^{1} \ldots\right.$ $\left.\int_{0}^{1}\left|\phi\left(t_{1}, t_{2}, \cdots, t_{n}\right)\right|^{2} d t_{1} d t_{2} \cdots d t_{n}\right]^{1 / 2}$.

EXAMPLE 2. Let $C$ consist of all real-valued continuous functions on $[0,1]$ which vanish at the origin. $C$ is a Banach space with the sup norm. Let $C^{\prime}=\left\{f \in C ; f\right.$ is absolutely continuous and $\left.f^{\prime} \in L^{2}(0,1)\right\} . \quad C^{\prime}$ is a Hilbert space with the inner product $\langle f, g\rangle=\int_{0}^{1} f^{\prime}(t) g^{\prime}(t) d t . \quad C^{\prime} \subset C$ is an abstract Wiener space [5;6 pp. 388-390]. Define $K: C^{\prime n} \rightarrow R$ by

$$
K\left(f_{1}, f_{2}, \cdots, f_{n}\right)=\int_{0}^{1} f_{1}^{\prime}(t) f_{2}(t) \cdots f_{n}(t) d t .
$$

Then $K$ is a Hilbert-Schmidt $n$-form on $C^{\prime}$ and it can be checked easily that $|K|_{2}=n^{-1 / 2}$. However, $K$ can not be extended to $C^{n}$. This example shows that $L^{n}(C ; R) \subsetneq L_{(2)}^{n}\left(C^{\prime} ; R\right)$.

Notation. Let $X$ be a Banach space. Let $S \in L^{n}(X ; R)$ and $T \in L(X ; X)$. Define the composition $S \circ_{j} T$ of $S$ and $T$ in the $j$-th factor by: $S \circ_{j} T\left(x_{1}, x_{2}, \cdots, x_{j}, \cdots, x_{n}\right)=S\left(x_{1}, x_{2}, \cdots, T x_{j}, \cdots, x_{n}\right), x_{k} \in X, k=1,2$, $\cdots, n$. Thus $S \circ_{j} T \in L^{n}(X ; R)$. $\|T\|_{X}$ denotes the operator norm of $T$.

LEMMA 1.2. (a) $\left\|S \circ_{j} T\right\| \leq\|S\|\|T\|_{B}, S \in L^{n}(B ; R), T \in L(B ; B)$.

(b) $\left|S \circ_{j} T\right| \leq|S|\|T\|_{H}, S \in L^{n}(H ; R), T \in L(H ; H)$.

(c) If $S \in L_{(2)}^{n}(H ; R)$ and $T \in L(H ; H)$ then $S \circ_{j} T \in L_{(2)}^{n}(H ; R)$ and $\left|S \circ_{j} T\right|_{2} \leq|S|_{2}\|T\|_{H}, j=1,2, \cdots, n$.

Proof. (a) and (b) are trivial. We use induction to prove (c). The cases with $n=1,2$ are well-known. Assume we have the lemma for $n-1$. Let $S \in L_{(2)}^{n}(H ; R)$ and $T \in L(H ; H)$. Clearly $\left(S \circ_{j} T\right)^{\sim}(h)=S^{\sim}(h) \circ_{j-1} T$ for $j=2,3, \cdots n$. Hence by induction $S \circ_{j} T \in L_{(2)}^{n}(H ; R), j=2,3, \cdots, n$. Furthermore, let $\left\{v_{k}\right\}$ be an orthonormal basis of $H$,

$$
\begin{aligned}
\left|S \circ_{j} T\right|_{2}^{2} & =\sum_{k}\left|\left(S \circ_{j} T\right)^{\sim}\left(v_{k}\right)\right|_{2}^{2} \\
& =\sum_{k}\left|S^{\sim}\left(v_{k}\right) \circ_{j-1} T\right|_{2}^{2} \\
& \leq \sum_{k}\left|S \sim\left(v_{k}\right)\right|_{2}^{2}\|T\|_{H}^{2} \quad \text { by induction } \\
& =|S|_{2}^{2}\|T\|_{H}^{2} .
\end{aligned}
$$


It remains to show the conclusion for $S \circ_{1} T$. But $\left(S \circ_{1} T\right)^{\sim}=S^{\sim} \circ T$. Thus $\left(S \circ_{1} T\right)^{\sim}(H) \subset S^{\sim}(H) \subset L_{(2)}^{n-1}(H ; R)$. Moreover by definition $\left|S \circ_{1} T\right|_{2}=$ the $H$-S-norm of $\left(S \circ_{1} T\right)^{\sim}=$ the $H$-S-norm of $S^{\sim} \circ T \leq$ the product of $H$ $S$-norm of $S^{\sim}$ and $\|T\|_{H}=|S|_{2}\|T\|_{H}$. Hence $\left|S \circ_{1} T\right|_{2} \leq|S|_{2}\|T\|_{H}$.

We have now various spaces $L^{n}(B ; R), L_{(2)}^{n}(H ; R)$ and $L^{n}(H ; R)$, $n \geq 1$. Each such space has three topologies, namely, the uniform topology, strong topology and weak topology. However, it can be shown, by a similar argument used in [9], that these topologies generate the same Borel field. Thus we do not need to specify the Borel field corresponding to a particular topology when we talk about the measurability of a random variable with values in those spaces.

Let $W(t)$ be a Wiener process in $B$. Let $\mathscr{M}_{t}$ be the $\sigma$-field generated by $\{W(s) ; 0 \leq s \leq t\}$. A stochastic process $\zeta(t, \omega), 0 \leq t$ ann $\omega \in \Omega$, is nonanticipating if it is $(t, \omega)$-jointly measurable and $\zeta(t, \cdot)$ is $\mathscr{M}_{t}$-measurable for each $t$. Let $\mathscr{L}\left[L_{(2)}^{n}(H ; R)\right]$ denote the space consisting of all nonanticipating stochastic processes $\xi(t)$ with state space $L_{(2)}^{n}(H ; R)$ such that $\int_{0}^{\tau} E|\xi(t)|_{2}^{2} d t<\infty$ for each $0<\tau<\infty$. We will define a linear operator $J$ from $\mathscr{L}\left[L_{(2)}^{n}(H ; R)\right]$ into $\mathscr{L}\left[L_{(2)}^{n-1}(H ; R)\right], n \geq 3$. (The cases $n=1,2$ have been defined in [14], $L_{(2)}^{0}(H ; R)=R$ by convention). In order to do this, we prove first a lemma about the space $\mathscr{L}\left[L^{n}(B ; R)\right]$ consisting of all non-anticipating stochastic processes $\zeta(t)$ with state space $L^{n}(B ; R)$ such that $\int_{0}^{\tau} E\|\zeta(t)\|^{2} d t<\infty$ for each $0<\tau<\infty$. By Lemma $1.1 \mathscr{L}\left[L^{n}(B ; R)\right]$ $\subset \mathscr{L}\left[L_{(2)}^{n}(H ; R)\right]$. Moreover, $\mathscr{L}\left[L^{n}(B ; R)\right]$ is dense in $\mathscr{L}\left[L_{(2)}^{n}(H ; R)\right]$ in the following sense:

LEMMA 1.3. If $\xi \in \mathscr{L}\left[L_{(2)}^{n}(H ; R)\right]$ then there exists a sequence $\xi_{n} \in$ $\mathscr{L}\left[L^{n}(B ; R)\right]$ such that $\int_{0}^{\tau} E\left|\xi_{n}(t)-\xi(t)\right|_{2}^{2} d t \rightarrow 0$ as $n \rightarrow \infty$ for each $0<\tau<\infty$.

LEMMA 1.4. If $\zeta \in \mathscr{L}\left[L^{n}(B ; R)\right]$ then

(a) for $s<t, E|\tilde{\zeta}(s)(W(t)-W(s))|_{2}^{2}=(t-s) E|\zeta(s)|_{2}^{2}$

(b) for $s<t<u<v, E\langle\tilde{\zeta}(s)(W(t)-W(s)), \tilde{\zeta}(u)(W(v)-W(u))\rangle_{2}=0$.

Remark. The special cases $n=1,2$ appeared in [14].

Proof. Let $\left\{Q_{k}\right\}$ be the projections given in the beginning of this section. Let

$$
\phi=|\tilde{\zeta}(s)(W(t)-W(s))|_{2}^{2}
$$


and

$$
\phi_{k}=\left|\tilde{\zeta}(s)\left(Q_{k}(W(t)-W(s))\right)\right|_{2}^{2} .
$$

Since $Q_{k}$ converges strongly to the identity in $B, \phi_{k} \rightarrow \phi$ almost surely. Furthermore,

$$
\begin{aligned}
\phi_{k} & \leq c^{2 n}\left\|\tilde{\zeta}(s)\left(Q_{k}(W(t)-W(s))\right)\right\|^{2} \quad \text { by Lemma } 1.1 \\
& \leq c^{2 n}\|\zeta(s)\|^{2}\left\|Q_{k}(W(t)-W(s))\right\|^{2} \\
& \left.\leq c^{2 n}\|\zeta(s)\|^{2}\left\|Q_{k}\right\|_{B}^{2} \| W(t)-W(s)\right) \|^{2} \\
& \leq \text { constant }\|\zeta(s)\|^{2}\|W(t)-W(s)\|^{2} .
\end{aligned}
$$

Recall that $\sup _{k}\left\|Q_{k}\right\|_{B}^{2}<\infty$ by the Uniform Boundedness Principle. But since $\zeta$ is non-anticipating,

$$
\begin{aligned}
E\left(\|\zeta(s)\|^{2}\|W(t)-W(s)\|^{2}\right) & =E\left(\|\zeta(s)\|^{2}\right) E\left(\|W(t)-W(s)\|^{2}\right) \\
& =E\left(\|\zeta(s)\|^{2}\right)(t-s) \int_{B}\|x\|^{2} p_{1}(d x),
\end{aligned}
$$

where $p_{1}$ is Wiener measure with variance parameter 1 . Therefore, by the Lebesgue dominated conyergence theorem,

$$
E|\tilde{\zeta}(s)(W(t)-W(s))|_{2}^{2}=\lim _{k \rightarrow \infty} E\left|\tilde{\zeta}(s)\left(Q_{k}(W(t)-W(s))\right)\right|_{2}^{2} .
$$

Without loss of generality, we may assume that $Q_{k}$ is the orthogonal projection onto the span of $\left\{f_{j} ; j=1,2, \cdots, k\right\}$, where $\left\{f_{j}\right\}$ is an orthonormal basis of $H$. Then

$$
\begin{aligned}
\mid \tilde{\zeta}(s)\left(Q_{k}\right. & (W(t)-W(s)))\left.\right|_{2} ^{2} \\
& =\left\langle\tilde{\zeta}(s)\left(Q_{k}(W(t)-W(s))\right), \tilde{\zeta}(s)\left(Q_{k}(W(t)-W(s))\right)\right\rangle_{2} \\
& =\sum_{j, m=1}^{k}\left(W(t)-W(s), f_{j}\right)\left(W(t)-W(s), f_{m}\right)\left\langle\tilde{\zeta}(s)\left(f_{j}\right), \tilde{\zeta}(s)\left(f_{m}\right)\right\rangle_{2} .
\end{aligned}
$$

Recall that $\zeta$ is non-anticipating and also that $E\left(W(t)-W(s), f_{j}\right)(W(t)-$ $\left.W(s), f_{m}\right)=(t-s) \delta_{j m}$. Hence we have

$$
E\left|\tilde{\zeta}(s)\left(Q_{k}(W(t)-W(s))\right)\right|_{2}^{2}=\sum_{j=1}^{k}(t-s) E\left|\tilde{\zeta}(s)\left(f_{j}\right)\right|_{2}^{2} .
$$

It follows from (4) and (5) that

$$
\begin{aligned}
E|\tilde{\zeta}(s)(W(t)-W(s))|_{2}^{2} & =\sum_{j=1}^{\infty}(t-s) E\left|\tilde{\zeta}(s)\left(f_{j}\right)\right|_{2}^{2} \\
& =(t-s) E|\zeta(s)|_{2}^{2} \quad \text { by (3). }
\end{aligned}
$$

Clearly, (b) can be shown in the same way. 
Now, we are ready to define the linear operator $J$ from $\mathscr{L}\left[L_{(2)}^{n}(H ; R)\right]$ into $\mathscr{L}\left[L_{(2)}^{n-1}(H ; R)\right]$. Let $\xi \in \mathscr{L}\left[L^{n}(B ; R)\right]$ be simple with jumps at $0<t_{1}$ $<t_{2}<\cdots<t_{k}$. Define, if $t_{j} \leq t<t_{j+1}, 0 \leq j \leq k$,

$$
\begin{gathered}
J_{\xi}(t)=\sum_{i=0}^{j-1} \tilde{\xi}\left(t_{i}\right)\left(W\left(t_{i+1}\right)-W\left(t_{i}\right)\right) \\
+\tilde{\xi}\left(t_{j}\right)\left(W(t)-W\left(t_{j}\right)\right) .
\end{gathered}
$$

Here $t_{0}=0$ and $t_{k+1}=\infty$ by convention. Clearly $J_{\xi} \in \mathscr{L}\left[L^{n-1}(B ; R)\right] \subset$ $\mathscr{L}\left[L_{(2)}^{n-1}(H ; R)\right]$. Without loss of generality we may assume that $t=t_{j}$ for some $j$. Thus

$$
J_{\xi}(t)=\sum_{i=0}^{j-1} \tilde{\xi}\left(t_{i}\right)\left(W\left(t_{i+1}\right)-W\left(t_{i}\right)\right) .
$$

Hence

$$
\left|J_{\xi}(t)\right|_{2}^{2}=\sum_{i, l=0}^{j-1}\left\langle\tilde{\xi}\left(t_{i}\right)\left(W\left(t_{i+1}\right)-W\left(t_{i}\right)\right), \tilde{\xi}\left(t_{l}\right)\left(W\left(t_{l+1}\right)-W\left(t_{l}\right)\right)\right\rangle_{2} .
$$

It follows immediately from Lemma 1.4 that

$$
\begin{aligned}
E\left|J_{\xi}(t)\right|_{2}^{2} & =\sum_{i=0}^{j-1}\left(t_{i+1}-t_{i}\right) E\left|\xi\left(t_{i}\right)\right|_{2}^{2} \\
& =E \int_{0}^{t}|\xi(s)|_{2}^{2} d s .
\end{aligned}
$$

Moreover, it is easy to see that

$$
E\left(J_{\xi}(t) \mid \mathscr{M}_{s}\right)=J_{\xi}(s) \quad s \leq t .
$$

From Lemma 1.3, (6), (7) and a standard argument in stochastic integral, we have

Proposition 1.1. There exists a linear operator $J$ from $\mathscr{L}\left[L_{(2)}^{n}(H ; R)\right]$ into $\mathscr{L}\left[L_{(2)}^{n-1}(H ; R)\right]$, denoted by $J_{\xi}(t)=\int_{0}^{t} \xi(s) d W(s)$, such that

(a) $J_{\xi}$ has continuous sample paths,

(b) $J_{\xi}$ is a martingale,

(c) $\operatorname{prob}\left\{\sup _{0 \leq t \leq \tau}\left|J_{\xi}(t)\right|_{2}>\delta\right\} \leq \delta^{-2} E\left|J_{\xi}(\tau)\right|_{2}^{2}$,

(d) $E J_{\xi}(t)=0$ and $E\left|J_{\xi}(t)\right|_{2}^{2}=E \int_{0}^{t}|\xi(s)|_{2}^{2} d s$.

Definition 2. Let $\mathscr{H}$ and $\mathscr{K}$ be two Hilbert spaces. A continuous bilinear map $S$ from $\mathscr{H} \times \mathscr{H}$ into $\mathscr{K}$ is said to be of trace-class-type if (i) for each $x \in \mathscr{K}, S_{x}$ is a trace class operator of $\mathscr{H}$, where $S_{x}(\cdot, \cdot)=$ $\langle S(\cdot, \cdot), x\rangle_{x}$ and (ii) the linear functional $x \rightarrow \operatorname{trace}_{\mathscr{H}} S_{x}$ is continuous. 
Notation. The definition implies obviously that there exists a unique element, denoted by TRACE $S$, of $\mathscr{K}$ such that $\langle\text { TRACE } S, x\rangle_{x}=\operatorname{trace}_{\mathscr{H}} S_{x}$ for all $x \in \mathscr{K} . \mathscr{S}(\mathscr{H} ; \mathscr{K})$ will denote the vector space of all trace-classtype bilinear maps from $\mathscr{H}$ into $\mathscr{K}$.

Proposition 1.2. (a) If $S \in \mathscr{S}(\mathscr{H} ; \mathscr{K})$ and $\left\{\phi_{k}\right\}$ is an orthonormal basis of $\mathscr{H}$ then $\sum_{k=1}^{\infty} S\left(\phi_{k}, \phi_{k}\right)$ converges in $\mathscr{K}$ to TRACE $S$,

(b) If $S \in \mathscr{S}(\mathscr{H} ; \mathscr{K})$ and $T, U \in L(\mathscr{H} ; \mathscr{H}), V \in L(\mathscr{K} ; \mathscr{K})$ then $S \circ[T \times U]$ and $V \circ S$ belong to $\mathscr{S}(\mathscr{H} ; \mathscr{K})$ and TRACE $V \circ S=V($ TRACE $S)$,

(c) $L^{2}\left(B ; L^{n}(B ; R)\right) \subset \mathscr{S}\left(H ; L_{(2)}^{n}(H ; R)\right)$.

Proof. (a) and (b) appeared in [15] in a similar form. (c) follows from the fact that $L^{2}(B ; R) \approx L\left(B ; B^{*}\right) \subset L_{1}(H ; H)$, the Banach space of all trace class operators of $H$ with the trace class norm $|\cdot|_{1}$. Actually, $|S|_{1} \leq\|S\| \int_{B}\|x\|^{2} p_{1}(d x)$ for all $S \in L^{2}(B ; R)$.

Notation. 1) If $T \in L^{n}(H ; R)$ and $S \in L\left(L^{n-1}(H ; R) ; L^{n-1}(H ; R)\right)$ we define the composition $S \triangle T$ of $S$ and $T$ to be an element of $L^{n}(H ; R)$ by $(S \Delta T)^{\sim}=S \circ \tilde{T}$. Thus $S \Delta T\left(h_{1}, h_{2}, \cdots, h_{n}\right)=S\left(\tilde{T}\left(h_{1}\right)\right)\left(h_{2}, \cdots, h_{n}\right)$.

2) If $S \in L^{n}(H ; R)$ and $T \in L\left(L^{n-1}(H ; R) ; R\right)$ we define $S \Delta T$ to be an element of $H$ by : $\langle S \Delta T, h\rangle=T(\tilde{S}(h)), h \in H$. Of course if $S \in L_{(2)}^{n}(H ; R)$ and $T \in L_{(2)}^{n-1}(H ; R)$ then define $\langle S \Delta T, h\rangle=\langle T, \tilde{S}(h)\rangle_{2}$.

Remarks. (1) If $T \in L_{(2)}^{n}(H ; R)$ and $L_{(2)}^{n-1}(H ; R)$ is invariant under $S$ then $S \Delta T \in L_{(2)}^{n}(H ; R)$.

(2) For the case $n=2$ in Notation 2, it is easy to see that $S \Delta h=$ $S^{*} h, h \in H$.

In [14] we proved an infinite dimensional analogue of well-known Ito's formula [8]. This formula was used in [17] to show the relation between the work of [14] and that of [19]. Later, in [15] we proved another version of Ito's formula and used it to construct diffusion processes in a Riemann-Wiener manifold. We will give three versions of Ito's formula for stochastic processes with state space $L^{n}(H ; R), n \geq 2$. Let $\mathscr{L}\left[L^{n}(H ; R)\right]$ consist of all non-anticipating processes $\zeta(t)$ with state space $L^{n}(H ; R)$ such that $\int_{0}^{\tau} E|\zeta(t)|^{2} d t<\infty$ for each $0<\tau<\infty$.

THEOREM 1. (Ito's formula). Let $\theta$ be a twice Frechet differentiable map from $L^{n}(H ; R)$ into itself such that for all $S \in L^{n}(H ; R)$ (i) $\theta^{\prime}(S)\left(L_{(2)}^{n}(H ; R)\right) \subset L_{(2)}^{n}(H ; R)$, (ii) $\theta^{\prime \prime}(S)\left(L_{(2)}^{n}(H ; R) \times L_{(2)}^{n}(H ; R)\right) \subset L_{(2)}^{n}(H ; R)$ 
and (iii) $\quad \theta^{\prime \prime}(S) \in \mathscr{S}\left(L_{(2)}^{n}(H ; R) ; L_{(2)}^{n}(H ; R)\right)$. If $\Phi(t)=\Phi_{0}+\int_{0}^{t} \xi(s) d W(s)+$ $\int_{0}^{t} \zeta(s) d s$, where $\xi \in \mathscr{L}\left[L_{(2)}^{n+1}(H ; R)\right]$ and $\zeta \in \mathscr{L}\left[L^{n}(H ; R)\right]$. Then

$$
\begin{aligned}
\theta(\Phi(t))= & \theta\left(\Phi_{0}\right)+\int_{0}^{t} \theta^{\prime}(\Phi(s)) \Delta \xi(s) d W(s)+\int_{0}^{t}\left\{\theta^{\prime}(\Phi(s))(\zeta(s))\right. \\
& \left.+\frac{1}{2} \operatorname{TRACE} \theta^{\prime \prime}(\Phi(s)) \circ[\tilde{\xi}(s) \times \tilde{\xi}(s)]\right\} d s .
\end{aligned}
$$

Proof. Kunita-Watanabe's method $[12 ; 13]$ can be employed here. We will sketch the outline only. Let $\varepsilon>0$ and $\left\{\sigma_{j}\right\}$ be an increasing sequence of stopping time converging to $\infty$ such that $\sigma_{0}=0$ and for $\sigma_{j} \leq s$, $t<\sigma_{j_{+1}}$, we have

$$
\left|\int_{s}^{t} \xi(\tau) d W(\tau)\right|_{2}<\varepsilon / 2
$$

and

$$
\left|\int_{s}^{t} \zeta(s) d s\right|<\varepsilon / 2
$$

Thus, whenever $\sigma_{j} \leq s, t<\sigma_{j+1}$

$$
|\Phi(t)-\Phi(s)|<\varepsilon .
$$

Because $\theta$ is twice Frechet differentiable, we have, whenever $x$ and $y$ are near in $L^{n}(H ; R)$,

$$
\theta(x)-\theta(y)=\theta^{\prime}(y)(x-y)+\frac{1}{2} \theta^{\prime \prime}(y)(x-y, x-y)+o\left(|x-y|^{2}\right) .
$$

Time parameter will be also subscribed from now on. Let $\tau_{j}=t \wedge \sigma_{j}$. Thus

$$
\begin{aligned}
\theta(\Phi(t))-\theta\left(\Phi_{0}\right)= & \sum_{j=1}^{\infty}\left[\theta\left(\Phi_{\tau_{j}}\right)-\theta\left(\Phi_{\tau_{j-1}}\right)\right] \\
= & \sum_{j=1}^{\infty} \theta^{\prime}\left(\Phi_{\tau_{j-1}}\right)\left(\Phi_{\tau_{j}}-\Phi_{\tau_{j-1}}\right) \\
& +\frac{1}{2} \sum_{j=1}^{\infty} \theta^{\prime \prime}\left(\Phi_{\tau_{j-1}}\right)\left(\Phi_{\tau_{j}}-\Phi_{\tau_{j-1}}, \Phi_{\tau_{j}}-\Phi_{\tau_{j-1}}\right) \\
& +o\left(\left|\Phi_{\tau_{j}}-\Phi_{\tau_{j-1}}\right|^{2}\right)
\end{aligned}
$$

Putting $\Phi_{\tau_{j}}-\Phi_{\tau_{j-1}}=\int_{\tau_{j-1}}^{\tau_{j}} \xi(s) d W(s)+\int_{\tau_{j-1}}^{\tau_{j}} \zeta(s) d s$ into the above equation, we see that to finish the proof it is sufficient to show the following two equalities : 


$$
\begin{array}{r}
\theta^{\prime}\left(\Phi_{s}\right)\left(\int_{s}^{t} \xi(\tau) d W(\tau)\right)=\int_{s}^{t} \theta^{\prime}\left(\Phi_{s}\right) \Delta \xi(\tau) d W(\tau) \\
\theta^{\prime \prime}\left(\Phi_{s}\right)\left(\int_{s}^{t} \xi(\tau) d W(\tau), \int_{s}^{t} \xi(\tau) d W(\tau)\right) \\
\quad=\int_{s}^{t} \operatorname{TRACE~} \theta^{\prime \prime}\left(\Phi_{s}\right) \circ[\tilde{\xi}(\tau) \times \tilde{\xi}(\tau)] d \tau
\end{array}
$$

(8) is easily checked, while (9) follows from the following observation: If $s \leq u<v$ then

$$
\begin{aligned}
& E \theta^{\prime \prime}\left(\Phi_{s}\right)(\tilde{\xi}(u)(W(v)-W(u)), \tilde{\xi}(u)(W(v)-W(u))) \\
& =(v-u) E \operatorname{TRACE} \theta^{\prime \prime}(\Phi(s)) \circ[\tilde{\xi}(u) \times \tilde{\xi}(u)] .
\end{aligned}
$$

If $s \leq u<v \leq u^{\prime}<v^{\prime}$ then

$$
E \theta^{\prime \prime}\left(\Phi_{s}\right)\left(\tilde{\xi}(u)(W(v)-W(u)), \tilde{\xi}\left(u^{\prime}\right)\left(W\left(v^{\prime}\right)-W\left(u^{\prime}\right)\right)\right)=0 .
$$

THEOREM 2 (Ito's formula). Let $\Gamma$ be a twice differentiable map from $L_{(2)}^{n}(H ; R)$ into itself such that $\Gamma^{\prime \prime}(S) \in \mathcal{P}\left(L_{(2)}^{n}(H ; R) ; L_{(2)}^{n}(H ; R)\right)$. If $\Phi(t)=\Phi_{0}+\int_{0}^{t} \xi(s) d W(s)+\int_{0}^{t} \zeta(s) d s$, where $\xi \in \mathscr{L}\left[L_{(2)}^{n+1}(H ; R)\right]$ and $\zeta \in \mathscr{L}$ $\left[L_{(2)}^{n}(H ; R)\right]$. Then

$$
\begin{aligned}
\Gamma(\Phi(t))= & \Gamma\left(\Phi_{0}\right)+\int_{0}^{t} \Gamma^{\prime}(\Phi(s)) \Delta \xi(s) d W(s) \\
& +\int_{0}^{t}\left\{\Gamma^{\prime}(\Phi(s))\left(\zeta(s)+\frac{1}{2} \operatorname{TRACE} \Gamma^{\prime \prime}(\Phi(s)) \circ[\tilde{\xi}(s) \times \tilde{\xi}(s)]\right\} d s .\right.
\end{aligned}
$$

THEOREM 3 (Ito's formula). Let $f$ be a twice Frechet differentiable function from $L^{n}(H ; R)$ (resp. $\left.L_{(2)}^{n}(H ; R)\right)$ into $R$. If $\Phi(t)=\Phi_{0}+\int_{0}^{t} \xi(s) d W(s)$ $+\int_{0}^{t} \zeta(s) d s$, where $\xi$ and $\zeta$ are same as Theorem 1 (resp. Theorem 2). Then

$$
\begin{aligned}
f(\Phi(t))= & f\left(\Phi_{0}\right)+\int_{0}^{t}\left(\xi(s) \Delta f^{\prime}(\Phi(s)), d W(s)\right) \\
& +\int_{0}^{t}\left\{f^{\prime}(\Phi(s))(\zeta(s))+\frac{1}{2} \operatorname{trace} f^{\prime \prime}(\Phi(s)) \circ[\tilde{\xi}(s) \times \tilde{\xi}(s)]\right\} d s .
\end{aligned}
$$

Remark. The proof of the above theorems goes in the same way as that of Theorem 1. We point out that $\xi(s) \Delta f^{\prime}(\Phi(s)$ ) (see Notation 2) following Proposition 1.2) is a non-anticipating process with state space $H$ and the stochastic integral $\int_{0}^{t}\left(\xi(s) \Delta f^{\prime}(\Phi(s))\right.$, $\left.d W(s)\right)$ was defined in the 
previous paper [14]. Furthermore, $f^{\prime \prime}(\Phi(s)) \circ[\tilde{\xi}(s) \times \tilde{\xi}(s)]$ is a non-anticipating process with state space $L_{1}(H ; H)$, the Banach space of all trace class operators of $H$. To see this, note that if $S \in L^{2}\left(L^{n}(H ; R) ; R\right)$ and $T \in L_{(2)}^{n+1}(H ; R)$ then $S \circ[\tilde{T} \times \tilde{T}]$ is a trace class operator of $H$.

THEOREM 4 (Girsanov-Skorokhod-McKean's formula). Suppose $\xi \in \mathscr{L}$ $\left[L_{(2)}^{3}(H ; R)\right]$ and $\eta \in \mathscr{L}\left[L^{2}(H ; R)\right]$ and with probability $1,\{\tilde{\xi}(t)(x), \eta(t)$; $0 \leq t<\infty, x \in H\}$ forms a commutative family of operators of $H\left(L^{2}(H ; R)\right.$ $\approx L(H ; H))$. Then the solution of

$$
Y(t)=I+\int_{0}^{t} Y(s) \circ_{3} \xi(s) * d W(s)+\int_{0}^{t} Y(s) \circ \eta(s) d s
$$

can be represented by

$$
Y(t)=\exp \left\{\int_{0}^{t} \xi(s) d W(s)+\int_{0}^{t}\left\{\eta(s)-\frac{1}{2} \operatorname{TRACE} \kappa \circ[\tilde{\xi}(s) \times \tilde{\xi}(s)]\right\} d s,\right.
$$

where $\kappa$ is the map from $L(H ; H) \times L(H ; H)$ into $L(H ; H)$ given by $\kappa(S, T)=S \circ T$.

Remark. We will discuss stochastic integral equation below. Moreover, in [16] we define, for $S \in L(H ; H)$ and $T \in L^{3}(H ; R), S \triangle T \in L^{3}(H ; R)$ by $(S \Delta T)^{\sim}(x)=S \circ(\tilde{T}(x)), x \in H$. It is easy to see that $S \Delta T$ is nothing but $T \circ_{3} S^{*}$. Thus equation (10) is the same as the equation in $\S 4$ of [16].

Proof. As in one dimensional case, we can solve (10) directly by using $\log$ function. Here, we prove this theorem in the reverse direction. Theorem 5 below implies that (10) has a unique solution. Thus it suffices to check that (11) satisfies (10). Consider the function $\theta(x)=$ $\exp (x), x \in L(H ; H) . \quad \theta$ is a $C^{\infty}$-function from $L(H ; H)$ into itself satisfying the hypothesis of Theorem 1 and, in particular, if $x$ and $y$ commute we have $\theta^{\prime}(x) y=e^{x} y$ and $\theta^{\prime \prime}(x)(y, y)=e^{x} y^{2}$. Let

$$
\Phi(t)=\int_{0}^{t} \xi(s) d W(s)+\int_{0}^{t}\left\{\eta(s)-\frac{1}{2} \operatorname{TRACE} \kappa \circ[\tilde{\xi}(s) \times \tilde{\xi}(s)]\right\} d s .
$$

Then $Y(t)=\exp \{\Phi(t)\}$. By stochastic differentiation given in Theorem 1, we have

$$
\begin{aligned}
d Y(t)= & \theta^{\prime}(\Phi(t)) \Delta \xi(t) d W(t)+\theta^{\prime}(\Phi(t))(\eta(t) \\
& \left.-\frac{1}{2} \operatorname{TRACE} \kappa \circ[\tilde{\xi}(t) \times \tilde{\xi}(t)]\right) d t \\
& +\frac{1}{2} \operatorname{TRACE~} \theta^{\prime \prime}(\Phi(t)) \circ[\tilde{\xi}(t) \times \tilde{\xi}(t)] d t
\end{aligned}
$$


Recall the notation 1) following Proposition 1.2. Let $h_{1}, h_{2}, h_{3} \in H$

$$
\begin{aligned}
\theta^{\prime}(\Phi(t)) & \Delta \xi(t)\left(h_{1}, h_{2}, h_{3}\right) \\
& =\theta^{\prime}(\Phi(t))\left(\tilde{\xi}(t) h_{1}\right)\left(h_{2}, h_{3}\right) \\
& =e^{\Phi(t)} \tilde{\xi}(t)\left(h_{1}\right)\left(h_{2}, h_{3}\right) \quad \text { by commutativity assumption, } \\
& =\left\langle Y(t) \tilde{\xi}(t)\left(h_{1}\right) h_{2}, h_{3}\right\rangle \\
& =\left\langle\tilde{\xi}(t)\left(h_{1}\right) h_{2}, Y(t)^{*} h_{3}\right\rangle \\
& =\xi(t)\left(h_{1}, h_{2}, Y(t)^{*} h_{3}\right) \\
& =\xi(t) \circ_{3} Y(t) *\left(h_{1}, h_{2}, h_{3}\right)
\end{aligned}
$$

Therefore, we have

$$
\theta^{\prime}(\Phi(t)) \triangle \xi(t)=\xi(t) \circ_{3} Y(t)^{*}
$$

Clearly,

$$
\theta^{\prime}(\Phi(t))(\eta(t))=Y(t) \circ \eta(t)
$$

Moreover, it can be checked easily that

$$
\theta^{\prime}(\Phi(t))\left(\kappa \circ[\tilde{\xi}(t) \times \tilde{\xi}(t)]=\theta^{\prime \prime}(\Phi(t)) \circ[\tilde{\xi}(t) \times \tilde{\xi}(t)] .\right.
$$

Putting (13), (14), and (15) into (12), we obtain

$$
d Y(t)=\xi(t) \circ_{3} Y(t)^{*} d W(t)+Y(t) \circ \eta(t) d t,
$$

or

$$
Y(t)=I+\int_{0}^{t} \xi(s) \circ_{3} Y(s) * d W(s)+\int_{0}^{t} Y(s) \circ \eta(s) d s .
$$

THEOREM 5. Let $f$ and $g$ be maps from $\left[t_{0}, \infty\right) \times L^{n}(H ; R) \times \Omega$ $\left(t_{0} \geq 0, n \geq 2\right)$ into $L_{(2)}^{n+1}(H ; R)$ and $L^{n}(H ; R)$, respectively. Assume that $f$ and $g$ satisfy the following conditions:

(a) for each $S \in L^{n}(H ; R), f(\cdot, S, \cdot)$ and $g(\cdot, S, \cdot)$ are non-anticipating,

(b) there is a constant $c$ such that with probability 1 ,

$$
|f(t, S)-f(t, T)|_{2}+|g(t, S)-g(t, T)| \leq c|S-T|,
$$

and

$$
|f(t, S)|_{2}+|g(t, S)| \leq c(1+|S|)
$$

for all $t \in\left[t_{0}, \infty\right)$ and $S, T \in L^{n}(H ; R)$. 
Let $\zeta \in \mathscr{L}\left[L^{n}(H ; R)\right]$ have continuous sample paths. Then the $L^{n}(H ; R)$ valued stochastic integral equation

$$
Y(t)=\zeta(t)+\int_{t_{0}}^{t} f(s, Y(s)) d W(s)+\int_{t_{0}}^{t} g(s, Y(s)) d s
$$

has a unique continuous solution $Y \in \mathscr{L}\left[L^{n}(H ; R)\right]$. Moreover, $Y(t)$ is a Markov process if $\zeta(t)$ is so.

Proof. We may assume that $t_{0} \leq t \leq t_{1}<\infty$. Let if be the Banach space of all non-anticipating processes $Y(t)$ in $L^{n}(H ; R)$ with norm

$$
\||Y|\|=\left\{\int_{t_{0}}^{t_{1}} E|Y(t)|^{2} d t\right\}^{1 / 2}<\infty .
$$

Clearly, $\mathscr{L}\left[L^{n}(H ; R)\right] \subset \mathfrak{U}$. Define a map $\Phi$ in $\mathfrak{A}$ by

$$
\Phi(Y)(t)=\zeta(t)+\int_{t_{0}}^{t} f(s, Y(s)) d W(s)+\int_{t_{0}}^{t} g(s, Y(s)) d s .
$$

It is easy to see that $\Phi$ is a map from $\mathfrak{A}$ into itself and $\Phi(Y)$ has continuous sample paths. Furthermore,

$$
E|\Phi(Y)(t)-\Phi(Z)(t)|^{2} \leq \alpha \int_{t_{0}}^{t} E|Y(s)-Z(s)|^{2} d s,
$$

where $\alpha$ is a constant depending only on $c, t_{0}$ and $t_{1}$. (16) implies that there exists an $N$ such that whenever $m \geq N$,

$$
\left\||| \Phi^{m}(Y)-\Phi^{m}(Z)\right\|\left|\leq \frac{1}{2}\|Y-Z\|\right| .
$$

The rest of the proof goes in the same way as Theorem 5.1 of [14].

THEOREM 6. In the hypothesis of Theorem 5 replace $L^{n}(H ; R)$ by $L_{(2)}^{n}(H ; R)$ and $L^{n}(H ; R)$-norm $|\cdot|$ by $L_{(2)}^{n}(H ; R)$-norm $|\cdot|_{2}$. Then the $L_{(2)}^{n}(H ; R)$-valued stochastic integral equation

$$
Z(t)=\zeta(t)+\int_{t_{0}}^{t} f(s, Z(s)) d W(s)+\int_{t_{0}}^{t} g(s, Z(s)) d s
$$

has a unique continuous solution $\left.\left.Z \in \mathscr{L}\left[L_{(2)}^{n}\right) H ; R\right)\right] . \quad Z(t)$ is a Markov process if $\zeta(t)$ is so.

Remark. Theorem 5 with $n=2$ and Theorem 6 with $n \geq 3$ will be used in the next section. Proof of Theorem 6 is obvious. 


\section{Regularity Properties}

We assume that $A$ and $\sigma$ satisfy the following conditions: $(A-1) \quad A$ is of the form $A(t, x)=C+K(t, x)$, where $C \in L(B ; B)$ and $K$ is a continuous map from $[0, \infty) \times B$ into $L_{(2)}(H ; H)$, $(A-2)$ There is a constant $\gamma$ such that for all $t \geq 0$ and $x, y \in B$,

$$
|K(t, x)-K(t, y)|_{2} \leq \gamma\|x-y\| \text { and }|K(t, x)|_{2} \leq \gamma(1+\|x\|),
$$

$(\sigma-1) \quad \sigma$ is continuous map from $[0, \infty) \times B$ into $B$ such that for all $t \geq 0$ and $x, y \in B,\|\sigma(t, x)-\sigma(t, y)\| \leq \gamma\|x-y\|$ and $\|\sigma(t, x)\| \leq \gamma(1+\|x\|)$.

Although the above conditions are weaker than those in Theorem 5.1 [14], it is easy to see that the proof there goes in the same way to conclude that under $(A-1),(A-2)$ and $(\sigma-1)$ the stochastic integral equation (1) has a unique non-anticipating continuous solution. Moreover, this solution is a Markov process. In the sequel, we denote this solution by $X_{x}(t)$, where $x$ is the starting point.

Definition 3 [7]. A map $f$ from $B$ into a Banach space $D$ is said to be Frechet differentiable at $x$ in $H$-directions (briefly, $H$-differentiable at $x$ ) if there exists a linear operator $T \in L(H ; D)$ such that $\| f(x+h)-$ $f(x)-T(h) \|_{D}=o(|h|), h \in H . \quad T$ is easily checked to be unique and will be denoted by $f^{\prime}(x)$, called the $H$-derivative of $f$ at $x . f$ is said to be $C_{H}^{1}$ if $f^{\prime}(x)$ exists for all $x \in B$ and $f^{\prime}$ is continuous from $B$ into $L(H ; D)$. Inductively, we can define $n$-th $H$-differentiability and $C_{H}^{n}$.

Notation. Let $D$ be a Banach space. $\mathscr{L}(D)$ will denote the Banach space of square integrable random variables taking values in $D$. Note that in Section 1 we used $\mathscr{L}[D]$ to denote the space of all non-anticipating processes $\zeta$ such that $\int_{0}^{\tau} E\|\zeta(t)\|_{D}^{2} d t<\infty$ for each $0 \leq \tau<\infty$.

DEFINITION 4. A function $\xi$ from $B$ into $\mathscr{L}(D)$ is said to be meansquare differentiable at $x$ in $H$-directions (briefly, $M S$ - $H$-differentiable at $x)$ if there is $\theta \in \mathscr{L}(L(H ; D))$ such that $E\|\xi(x+h)-\xi(x)-\theta(h)\|_{D}^{2}=$ $o\left(|h|^{2}\right), h \in H . \quad \theta$ is unique and will be denoted by $\delta \xi_{x}$, called the $M S-H$ derivative of $\xi$ at $x$. $M S-H$-differentiability and $M S-C_{H}^{n}(n \geq 1)$ are defined in an obvious way.

DEFINITION 5. A transformation $Z$ from $B$ into $\mathscr{L}[D]$ is said to be $M S-H$-differentiable if there is a transformation $Y$ from $B$ into 
$\mathscr{L}[L(H ; D)]$ such that for each $t \geq 0, Y(t)$ is the $M S$ - $H$-derivative of $Z(t)$. $Y$ is unique and will be denoted by $\delta Z$. Higher order $M S$ - $H$-derivatives will be denoted by $\delta^{n} Z, n \geq 2$.

EXAMPLE 1. Let $X(t)=x+W(t)$, where $W$ is Wiener process starting at the origin. Then $\delta X_{x}(t)=I$ for all $x$ and $\delta^{n} X_{x}(t) \equiv 0, n \geq 2$.

ExAmPLE 2. Consider the Langevin equation $d U(t)=d W(t)-U(t) d t$. Its solution $U(t)$ is called Uhlenbeck-Ornstein process. We have $\delta U_{x}(t)$ $=e^{-t} I$ for all $x, \delta^{n} U_{x}(t) \equiv 0, n \geq 2$.

EXAMPLE 3. Let $K \in L_{(2)}(H ; H), T \in L(B ; H)$ and $x_{0} \in H \cap \operatorname{ker} T^{*}$, where $*$ denotes the adjoint of operators of $H$. Consider the equation $d X(t)=(I+K) d W(t)+f\left(|T X(t)|^{2}\right) x_{0} d t$, where $f$ is a real-valued differentiable function with compact support. We have $\delta X_{x}(t)=e^{\tau_{x}(t)}$, where $\zeta_{x} \in \mathscr{L}[L(H ; H)]$ is given by $\zeta_{x}(t)=2\left[\int_{0}^{t} f^{\prime}\left(\left|T X_{x}(s)\right|^{2}\right)\left\langle T^{2} X_{x}(s), \cdot\right\rangle d s\right] x_{0}$.

Remark. Two transformations $Z_{1}$ and $Z_{2}$ from $B$ into $\mathscr{L}[D]$ have the same $M S-H$-derivative if and only if there exists $\xi \in \mathscr{L}[D]$ such that $Z_{1}-Z_{2} \equiv \xi$. Moreover, if $\xi$ is an $M S-H$-differentiale function from $B$ into $\mathscr{L}(D)$ then $\xi_{x+h}-\xi_{x}=\int_{0}^{1} \delta \xi_{x+\tau h}(h) d \tau, x \in B$ and $h \in H$.

Proposition 2.1. Suppose $\xi \in \mathscr{L}\left[L_{(2)}^{n+1}(H ; R)\right], n \geq 0$. Then $E\left|J_{\xi}(t)\right|_{2}^{4}$ $=2 \int_{0}^{t} E\left[\left|J_{\xi}(s)\right|_{2}^{2}|\xi(s)|_{2}^{2}\right] d s+4 \int_{0}^{t} E\left|\xi(s) \Delta J_{\xi}(s)\right|^{2} d s$.

Remark. $\xi \Delta J_{\xi} \in \mathscr{L}[H]$. See Notation 2) following Proposition 1.2.

Proof. Apply Ito's formula in Theorem 3 to the function $f(x)=$ $|x|_{2}^{4}, x \in L_{(2)}^{n}(H ; R)$ and to the process $J_{\xi}(t)=\int_{0}^{t} \xi(s) d W(s), \xi \in \mathscr{L}\left[L_{(2)}^{n+1}(H ; R)\right]$. Note that $f^{\prime}(x)=4|x|_{2}^{2}\langle x, \cdot\rangle_{2}$ and $f^{\prime \prime}(x)=4|x|_{2}^{2}\langle\cdot, \cdot\rangle_{2}+8\langle x, \cdot\rangle_{2}\langle x, \cdot\rangle_{2}$. Hence we have

$$
\begin{aligned}
f\left(J_{\xi}(t)\right)= & \int_{0}^{t}\left(\xi(s) \Delta f^{\prime}\left(J_{\xi}(s)\right), d W(s)\right) \\
& +\frac{1}{2} \int_{0}^{t} \operatorname{trace} f^{\prime \prime}\left(J_{\xi}(s)\right) \circ[\tilde{\xi}(s) \times \tilde{\xi}(s)] d s .
\end{aligned}
$$

After taking expectation, we get

$$
E\left|J_{\xi}(t)\right|_{2}^{4}=\frac{1}{2} \int_{0}^{t} E \text { trace } f^{\prime \prime}\left(J_{\xi}(s)\right) \circ[\tilde{\xi}(s) \times \tilde{\xi}(s)] d s .
$$


Let $\left\{e_{i}\right\}$ be an orthonormal basis of $H$, then

$$
\begin{aligned}
& \operatorname{trace}\left|J_{\xi}(s)\right|_{2}^{2}\langle\tilde{\xi}(s), \tilde{\xi}(s)\rangle_{2} \\
& =\sum_{j}\left|J_{\xi}(s)\right|_{2}^{2}\left\langle\tilde{\xi}(s) e_{j}, \tilde{\xi}(s) e_{j}\right\rangle_{2} \\
& =\left|J_{\xi}(s)\right|_{2}^{2}|\xi(s)|_{2}^{2},
\end{aligned}
$$

and

$$
\begin{aligned}
\operatorname{trace} & \left\langle J_{\xi}(s), \tilde{\xi}(s)\right\rangle_{2}\left\langle J_{\xi}(s), \tilde{\xi}(s)\right\rangle_{2} \\
& =\sum_{j}\left\langle J_{\xi}(s), \tilde{\xi}(s) e_{j}\right\rangle_{2}^{2} \\
& =\sum_{j}\left\langle\xi(s) \Delta J_{\xi}(s), e_{j}\right\rangle^{2} \\
& =\left|\xi(s) \Delta J_{\xi}(s)\right|^{2} .
\end{aligned}
$$

Combining (17), (18), and (19), we obtain the conclusion.

Proposition 2.2. Suppose $\xi \in \mathscr{L}\left[L_{(2)}^{n+1}(H ; R)\right], n \geq 0$. Then $E\left|J_{\xi}(t)\right|_{2}^{4}$ $\leq 36 t \int_{0}^{t} E|\xi(s)|_{2}^{4} d s$.

Proof. First note that from the previous proposition $E\left|J_{\xi}(t)\right|_{2}^{4}$ is an increasing function of $t$. Hence

$$
\begin{aligned}
E\left[\left|J_{\xi}(s)\right|_{2}^{2}|\xi(s)|_{2}^{2}\right] & \left.\leq\left\{E\left|J_{\xi}(s)\right|_{2}^{4}\right\}^{1 / 2} E|\xi(s)|_{2}^{4}\right\}^{1 / 2} \\
& \leq\left\{E\left|J_{\xi}(t)\right|_{2}^{4}\right\}^{1 / 2}\left\{E|\xi(s)|_{2}^{4}\right\}^{1 / 2}
\end{aligned}
$$

Now, recall that for $S \in L_{(2)}^{n+1}(H ; R)$ and $T \in L_{(2)}^{n}(H ; R), S \Delta T$ is an element in $H$ defined by $\langle S \Delta T, h\rangle=\langle T, \tilde{S}(h)\rangle_{2}$. Thus we have $|S \Delta T| \leq|T|_{2}|S|_{2}$. So,

$$
\begin{aligned}
E\left|\xi(s) \Delta \dot{J}_{\xi}(s)\right|^{2} & \leq E\left[|\xi(s)|_{2}^{2}\left|J_{\xi}(s)\right|_{2}^{2}\right] \\
& \leq\left\{E\left|J_{\xi}(t)\right|_{2}^{4}\right\}^{1 / 2}\left\{E|\xi(s)|_{2}^{4}\right\}^{1 / 2} .
\end{aligned}
$$

Therefore, by the previous proposition

$$
\begin{aligned}
E\left|J_{\xi}(t)\right|_{2}^{4} & \leq 6\left\{E\left|J_{\xi}(t)\right|_{2}^{4}\right\}^{1 / 2} \int_{0}^{t}\left\{E|\xi(s)|_{2}^{4}\right\}^{1 / 2} d s \\
& \leq 6\left\{E\left|J_{\xi}(t)\right|_{2}^{4}\right\}^{1 / 2}\left\{t \int_{0}^{t} E|(s)|_{2}^{4} d s\right\}^{1 / 2},
\end{aligned}
$$

and

$$
E\left|J_{\xi}(t)\right|_{2}^{4} \leq 36 t \int_{0}^{t} E|\xi(s)|_{2}^{4} d s .
$$

Notation. 1) Let $S \in L^{n}(H ; R) . \quad \hat{S} \in L^{n-1}(H ; H)$ is defined by $\left\langle\hat{S}\left(h_{1}\right.\right.$, 
$\left.\left.h_{2}, \cdots, h_{n-1}\right), h\right\rangle=S\left(h, h_{1}, h_{2}, \cdots, h_{n-1}\right) . \quad$ Note that for $n=2, \tilde{S}=S$, while $\hat{S}=S^{*}$.

2) Let $T \in L^{3}(H ; R)$ and $S \in L^{n}(H ; R)$. Define $S: T \in L^{n+1}(H ; R)$ by $S: T\left(h_{1}, h_{2}, \cdots, h_{n}, h_{n+1}\right)=T\left(h_{1}, \hat{S}\left(h_{2}, \cdots, h_{n}\right), h_{n+1}\right)$. Note that if $T \in L_{(2)}^{3}$ $(H ; R)$ and $S \in L_{(2)}^{n}(H ; R)$ then $S: T \in L_{(2)}^{n+1}(H ; R)$ and $|S: T|_{2} \leq|S|_{2}|T|_{2}$. But for $n=2,|S: T|_{2} \leq|S||T|_{2}$.

Remark. If $T \in L^{3}(H ; R), S \in L^{2}(H ; R)$ and $\tilde{T}(h)$ commutes with $S$ for all $h \in H$. Then $S: T=T \circ_{3} S$.

ThEOREM 7. Assume $A$ and $\sigma$ satisfy $(A-1),(A-2),(\sigma-1)$ and the following conditions:

$(A-3) \quad K(t, x)$ is $C_{H}^{2}$ in $x$ variable with $K^{\prime}(t, x) \in L_{(2)}^{3}(H ; R)$ and $K^{\prime \prime}(t, x) \in L_{(2)}^{4}(H ; R) . \quad K^{\prime}(\cdot, \cdot)$ and $K^{\prime \prime}(\cdot, \cdot)$ are bounded continuous maps from $[0, \tau) \times B$ into $L_{(2)}^{3}(H ; R)$ and $L_{(2)}^{4}(H ; R)$, respectively, for each $\tau$. $(A-4)$ for all $t$ and $x, K^{\prime}(t, x) \in L_{(2)}^{3}(H ; R)$ and $K^{\prime \prime}(t, x) \in L_{(2)}^{4}(H ; R)$ are symmetric in the first two components,

$(\sigma-2) \quad \sigma(t, x)$ ir $C_{H}^{2}$ in $x$ variable with $\sigma^{\prime}(t, x) \in L(H ; H)$ and $\sigma^{\prime \prime}(t, x) \in$ $L_{(2)}^{3}(H ; R) . \quad \sigma^{\prime}(\cdot, \cdot)$ and $\sigma^{\prime \prime}(\cdot, \cdot)$ are bounded continuous maps from $[0, \tau)$ $\times B$ into $L(H ; H)$ and $L_{(2)}^{3}(H ; R)$ respectively, for each $\tau$. Then the diff $u$ sion process given by the solution of the stochastic integral equation

$$
X(t)=X(0)+\int_{0}^{t} A(s, X(s)) d W(s)+\int_{0}^{t} \sigma(s, X(s)) d s
$$

is twice MS-H-differentiable. The first derivative at $x$ is given by the solution of the operator-valued stochastic integral equation

$$
Y(t)=I+\int_{0}^{t} Y(s): K^{\prime}\left(s, X_{x}(s)\right) d W(s)+\int_{0}^{t} \sigma^{\prime}\left(s, X_{x}(s)\right) \circ Y(s) d s .
$$

The second derivative at $x$ is given by the solution of the 3-form-valued stochastic integral equation

$$
Z(t)=\phi(t)+\int_{0}^{t} Z(s): K^{\prime}\left(s, X_{x}(s)\right) d W(s)+\int_{0}^{t} Z(s) \circ_{3} \sigma^{\prime \prime}\left(s, X_{x}(s)\right)^{*} d s,
$$

where

$$
\phi(t)=\int_{0}^{t} K^{\prime \prime}\left(s, X_{x}(s)\right) \circ_{4}\left[\delta X_{x}(s)^{2}\right]^{*} d W(s)+\int_{0}^{t} \hat{\sigma}^{\prime \prime}\left(s, X_{x}(s)\right) \circ\left[\delta X_{x}(s) \times \delta X_{x}(s)\right] d s .
$$

Furthermore, $\delta X_{x} \in \mathscr{L}[L(H ; H)]$ and $\delta^{2} X_{x} \in \mathscr{L}\left[L_{(2)}^{3}(H ; R)\right]$. 
Proof. We need to show that $E\left\|X_{x+h}(t)-X_{x}(t)-Y(t) h\right\|^{2}=o\left(|h|^{2}\right)$, $h \in H$. But it is easy to see that $X_{x+h}(t)-X_{x}(t)$ is in $H$. Thus we will show below a stronger statement, namely, $E\left|X_{x+h}(t)-X_{x}(t)-Y(t) h\right|^{2}=$ $o\left(|h|^{2}\right), h \in H$. Assume $0 \leq t \leq \tau$. Let $\psi_{h}(t)=X_{x+h}(t)-X_{x}(t)$. Then

$$
\psi_{h}(t)=h+\int_{0}^{t} \xi_{h}(s)\left(\psi_{h}(s)\right) d W(s)+\int_{0}^{t} \zeta_{h}(s)\left(\psi_{h}(s)\right) d s,
$$

where $\xi_{h}(s)$ and $\zeta_{h}(s)$ are given by

$$
\begin{gathered}
\xi_{h}(s)=\int_{0}^{1} A^{\prime}\left(s, X_{x}(s)+\tau \psi_{h}(s)\right) d \tau=\int_{0}^{1} K^{\prime}\left(s, X_{x}(s)+\tau \psi_{h}(s)\right) d \tau, \\
\zeta_{h}(s)=\int_{0}^{1} \sigma^{\prime}\left(s, X_{x}(s)+\tau \psi_{h}(s)\right) d s .
\end{gathered}
$$

On the other hand,

$$
Y(t) h=h+\int_{0}^{t} K^{\prime}\left(s, X_{x}(s)\right)(Y(s) h) d W(s)+\int_{0}^{t} \sigma^{\prime}\left(s, X_{x}(s)\right)(Y(s) h) d s .
$$

Here we have used the condition $(A-4)$ to bring $h$ into the integral sign.

Now, it can be shown with some computation that

$$
\begin{aligned}
E \mid \xi_{h}(s)\left(\psi_{h}(s)\right) & -\left.K^{\prime}\left(s, X_{x}(s)\right)(Y(s) h)\right|_{2} ^{2} \\
& \leq c_{1} E\left|\psi_{h}(s)-Y(s) h\right|^{2}+c_{2} E\left(\left|\psi_{h}(s)\right|^{2}|Y(s) h|^{2}\right),
\end{aligned}
$$

and

$$
\begin{aligned}
E \mid \zeta_{h}(s)\left(\psi_{h}(s)\right) & -\left.\sigma^{\prime}\left(s, X_{x}(s)\right)(Y(s) h)\right|^{2} \\
& \leq c_{1} E\left|\psi_{h}(s)-Y(s) h\right|^{2}+c_{2} E\left(\left|\psi_{h}(s)\right|^{2}|Y(s) h|^{2}\right),
\end{aligned}
$$

where $c_{1}$ and $c_{2}$ are constants independent of $s$ and $t$. From (23)-(28), we obtain for all $0 \leq t \leq \tau$,

$$
E\left|\psi_{h}(t)-Y(t) h\right|^{2} \leq c_{3} \lambda(h)+c_{4} \int_{0}^{t} E\left|\psi_{h}(s)-Y(s) h\right|^{2} d s,
$$

where $c_{3}$ and $c_{4}$ are constants independent of $t$, and

$$
\lambda(h)=\int_{0}^{\tau} E\left|\psi_{h}(s)\right|^{2}|Y(s) h|^{2} d s .
$$

Hence by Gronwall's Lemma,

$$
E\left|\psi_{h}(t)-Y(t) h\right|^{2} \leq c_{3} \lambda(h) e^{c_{4} \tau} \quad 0 \leq t \leq \tau .
$$


But $\lambda(h) \leq|h|^{2} \int_{0}^{\tau} E\left|\psi_{h}(s)\right|^{2}\|Y(s)\|_{H}^{2} d s$, hence we are remained to prove that

$$
\lim _{|h| \rightarrow 0} \int_{0}^{\tau} E\left|\psi_{h}(s)\right|^{2}\|Y(s)\|_{H}^{2} d s=0 .
$$

By a complicated computation using Proposition 2.2 and Gronwall's Lemma, we have

$$
E\left|\psi_{h}(t)\right|^{4} \leq \mathrm{constant}|h|^{4}, \quad 0 \leq t \leq \tau,
$$

and

$$
E\|Y(t)\|_{H}^{4} \leq \text { constant }, \quad 0 \leq t \leq \tau .
$$

Hence (29) is evident and, in particular, we have also that $Y \in \mathscr{L}[L(H ; H)]$.

We should not try to prove the second assertion. But we will show that $\phi$ given in $(22)$ is in $\mathscr{L}\left[L_{(2)}^{3}(H ; R)\right] . \quad \phi$ is clearly non-anticipating.

$$
\begin{aligned}
|\phi(t)|_{2}^{2} & \leq 2 \mid \int_{0}^{t} K^{\prime \prime}\left(s,\left.X_{x}(s) \circ_{4}\left[\delta X_{x}(s)^{2}\right]^{*} d W(s)\right|_{2} ^{2}\right. \\
& +2\left|\int_{0}^{t} \hat{\sigma}^{\prime \prime}\left(s, X_{x}(s)\right) \circ\left[\delta X_{x}(s) \times \delta X_{x}(s)\right] d s\right|_{2}^{2} .
\end{aligned}
$$

Apply Proposition 1.1 to get

$$
\begin{aligned}
E\left|\int_{0}^{t} K^{\prime \prime}\left(s, X_{x}(s)\right) \circ_{4}\left[\delta X_{x}(s)^{2}\right]^{*} d W(s)\right|_{2}^{2} \\
\quad=\int_{0}^{t} E \mid K^{\prime \prime}\left(s,\left.X_{x}(s) \circ_{4}\left[\delta X_{x}(s)^{2}\right]^{*}\right|_{2} ^{2} d s\right. \\
\quad \leq \int_{0}^{t} E\left\|\delta X_{x}(s)\right\|_{H}^{2}\left|K^{\prime \prime}\left(s, X_{x}(s)\right)\right|_{2}^{2} d s \\
\quad \leq \alpha \int_{0}^{t} E\left\|\delta X_{x}(s)\right\|_{H}^{2} d s
\end{aligned}
$$

where $\alpha=\sup _{0 \leq s \leq r, x \in B}\left|K^{\prime \prime}(s, x)\right|_{2}^{2}<\infty$.

On the other hand,

$$
\begin{aligned}
E \mid \int_{0}^{t} \hat{\sigma}^{\prime \prime} & \left.\left(s, X_{x}(s)\right) \circ\left[\delta X_{x}(s) \times \delta X_{x}(s)\right] d s\right|_{2} ^{2} \\
& \leq t \int_{0}^{t} E\left|\hat{\sigma}^{\prime \prime}\left(s, X_{x}(s)\right) \circ\left[\delta X_{x}(s) \times \delta X_{x}(s)\right]\right|_{2}^{2} d s \\
& \leq t \int_{0}^{t} E\left|\sigma^{\prime \prime}\left(s, X_{x}(s)\right)\right|_{2}^{2}\left\|\delta X_{x}(s)\right\|_{H}^{2} d s \\
& \leq \beta t \int_{0}^{t} E\left\|\delta X_{x}(s)\right\|_{H}^{2} d s
\end{aligned}
$$


where $\beta=\sup _{0 \leq s \leq \tau, x \in B}\left|\sigma^{\prime \prime}\left(s, X_{x}(s)\right)\right|_{2}^{2}<\infty$.

Note that we have used the property that if $S \in L_{(2)}^{3}(H ; R)$ and $T \in L(H ; H)$ then $\hat{S} \circ[T \times T] \in L_{(2)}^{3}(H ; R)$ and $|\hat{S} \circ[T \times T]|_{2} \leq|S|_{2}\|T\|_{H}^{2}$. This can be seen by observing that $\hat{S} \circ[T \times T]=\left(S \circ_{1} T\right) \circ_{2} T$ and then applying Lemma 1.2. (30), (31) and (32) clearly show that $\int_{0}^{\tau} E|\phi(t)|_{2}^{2} d t<\infty$ for each $0 \leq \tau<\infty$. Hence $\phi \in \mathscr{L}\left[L_{(2)}^{3}(H ; R)\right]$.

THEOREM 8. Assume $A$ and $\sigma$ satisfy $(A-1),(A-2),(\sigma-1)$ and the following conditions:

$(A-3)^{*} \quad K(t, x)$ is $C_{H}^{n}(n \geq 2)$ in $x$ variable with $K^{(j)}(t, x) \in L_{(2)}^{j+2}(H ; R)$, $j=1,2, \cdots, n . \quad K^{(j)}$ is bounded and continuous from $[0, \tau) \times B$ into $L_{(2)}^{j+2}(H ; R)$ for each $0 \leq \tau<\infty, j=1,2, \cdots, n$.

$(A-4)^{*}$ for all $t$ and $x, K^{(j)}(t, x) \in L_{(2)}^{j+2}(H ; R)$ is symmetric in the first two components, $j=1,2, \cdots, n$.

$(\sigma-2)^{*} \quad \sigma(t, x)$ is $C_{H}^{n}(n \geq 2)$ in $x$ variable with $\sigma^{\prime}(t, x) \in L(H ; H)$ and $\sigma^{(j)}(t, x) \in L_{(2)}^{j+1}(H ; R), j=2,3, \cdots, n . \quad \sigma^{\prime}$ and $\sigma^{(j)}$ are bounded, continuous from $[0, \tau) \times B$ into $L(H ; H)$ and $L_{(2)}^{j+1}(H ; R)$, respectively, for each $0 \leq \tau$ $<\infty, j=2,3, \cdots, n$.

Then the diffusion process $X(t)$ given by the solution of the equation

$$
X(t)=X(0)+\int_{0}^{t} A(s, X(s)) d W(s)+\int_{0}^{t} \sigma(s, X(s)) d s
$$

is $n$-th $M S$-H-differentiable. Furthermore, $\delta X \in \mathscr{L}[L(H ; H)]$ and $\delta^{j} X \in \mathscr{L}$ $\left[L_{(2)}^{j+1}(H ; R)\right], j=2,3, \cdots, n$.

THEOREM 9. Suppose $A$ and $\sigma$ satisfy the conditions $(A-1),(A-2)$, $(A-3)^{*},(A-4)^{*},(\sigma-1)$ and $(\sigma-2)^{*}$. Let $X(t)$ be the diffusion process given by the diffusion coefficients $A$ and $\sigma$. If $f$ is a $C_{H}^{k}$-function in $B$ with bounded derivatives, $0 \leq k \leq n$, then the function $\theta(x)=E_{x}[f(X(t))]$ is also $C_{H}^{k}$. Its first two $H$-derivatives are

$$
\begin{gathered}
\theta^{\prime}(x)=E\left[\delta X_{x}(t)^{*}\left(f^{\prime}\left(X_{x}(t)\right)\right)\right], \\
\theta^{\prime \prime}(x)=E\left\{\delta^{2} X_{x}(t)^{\vee}\left(f^{\prime}\left(X_{x}(t)\right)\right)+f^{\prime \prime}\left(X_{x}(t)\right) \circ\left[\delta X_{x}(t) \times \delta X_{x}(t)\right]\right\} .
\end{gathered}
$$

Moreover, $\theta^{\prime \prime}(x)$ is a Hilbert-Schmidt operator of $H$ for all $x \in B$ if $f^{\prime \prime}$ is so.

Notation. If $S \in L^{n}(H ; R)$ then $S^{\sim} \in L\left(H ; L^{n-1}(H ; R)\right)$ is defined to be $\check{S}(h)=S(\cdot, \cdot, \cdots, \cdot, h)$. Note that if $S \in L_{(2)}^{n}(H ; R)$ then $\check{S}(h) \in L_{(2)}^{n-1}(H ; R)$ 
and $\check{S}$ is a Hilbert-Schmidt operator from $H$ into $L_{(2)}^{n-1}(H ; R)$.

Proof. Let $\psi_{h}(t)=X_{x+h}(t)-X_{x}(t)$. Then

$$
\begin{aligned}
f\left(X_{x+h}(t)\right) & -f\left(X_{x}(t)\right) \\
= & \int_{0}^{1}\left\langle f^{\prime}\left(X_{x}(t)+\tau \psi_{h}(t)\right), \psi_{h}(t)\right\rangle d \tau .
\end{aligned}
$$

Hence

$$
\begin{aligned}
& f\left(X_{x+h}(t)\right)-f\left(X_{x}(t)\right)-\left\langle\delta X_{x}(t)^{*}\left(f^{\prime}\left(X_{x}(t)\right)\right), h\right\rangle \\
& =\int_{0}^{1}\left\langle f^{\prime}\left(X_{x}(t)+\tau \psi_{h}(t)\right)-f^{\prime}\left(X_{x}(t)\right), \psi_{h}(t)\right\rangle d \tau \\
& \quad+\left\langle f^{\prime}\left(X_{x}(t)\right), \psi_{h}(t)-\delta X_{x}(t) h\right\rangle=\alpha(h)+\beta(h) .
\end{aligned}
$$

Obviously, $E|\beta(h)|=o(|h|)$ since $f^{\prime}$ is bounded and $X(t)$ is $M S$ - $H$-differentiable.

On the other hand,

$$
\begin{aligned}
E|\alpha(h)| & \leq \int_{0}^{1} E\left|f^{\prime}\left(X_{x}(t)+\tau \psi_{h}(t)\right)-f^{\prime}\left(X_{x}(t)\right)\right|\left|\psi_{h}(t)\right| d \tau \\
& \leq\left\{E\left|\psi_{h}(t)\right|^{2}\right\}^{1 / 2} \int_{0}^{1}\left\{E\left|f^{\prime}\left(X_{x}(t)+\tau \psi_{h}(t)\right)-f^{\prime}\left(X_{x}(t)\right)\right|^{2}\right\}^{1 / 2} d \tau \\
& \leq c|h|\left\{\int_{0}^{1} E\left|f^{\prime}\left(X_{x}(t)+\tau \psi_{h}(t)\right)-f^{\prime}\left(X_{x}(t)\right)\right|^{2} d \tau\right\}^{1 / 2},
\end{aligned}
$$

where $c$ is a constant independent of $h$. Apply Lebesgue's dominated convergence theorem to conclude that $E|\alpha(h)|=o(|h|)$. Therefore,

$$
E\left|f\left(X_{x+h}(t)\right)-f\left(X_{x}(t)\right)-\left\langle\delta X_{x}(t)^{*}\left(f^{\prime}\left(X_{x}(t)\right)\right), h\right\rangle\right|=o(|h|), \quad h \in H .
$$

This proves (33). (34) can be proved in a similar way. Furthermore, $\theta^{(j)}(x)(3 \leq j \leq k)$ can be expressed by using the first $j$-th derivatives of $f$ and $X(t)$. Finally $\theta^{\prime \prime}(x)$ is a Hilbert-Schmidt operator by the remark in Notation above and by the property: if $S \in L_{(2)}^{2}(H ; R)$ and $T \in L(H ; H)$ then $S \circ[T \times T] \in L_{(2)}^{2}(H ; R)$. In fact, $S \circ[T \times T]=\left(S \circ_{1} T\right) \circ_{2} T$, hence $|S \circ[T \times T]|_{2} \leq|S|_{2}\|T\|_{H}^{2}$ by Lemma 1.2

To finish this paper, we consider the homogeneous case, i.e. $A$ and $\sigma$ are independent of $t . \quad A$ and $\sigma$ satisfy $(A-1),(A-2)$ and $(\sigma-1)$. In this case $X(t)$ generates a semi-group $\left\{P_{t} ; t \geq 0\right\}, P_{t} f(x)=E_{x}[f(X(t))]$. Let $C_{0}$ be the Banach space of bounded continuous functions on $B$ vanishing at infinity. $C_{0}$ has the sup norm. Assume the $B$-norm $\|\cdot\|^{2}$ is $C_{H}^{2}$ 
such that its second $H$-derivative has bounded range in $L_{1}(H ; H)$.

THEOREM 10. The operators $P_{t}, t \geq 0$, form a strongly continuous contraction semi-group on $C_{0}$.

Proof*. $P_{t}, t \geq 0$ are obviously strongly continuous and contractive. We need only to show that $P_{t} f \in C_{0}$ whenever $f \in C_{0}$.

Let $\theta(x)=\log \left(1+\|x\|^{2}\right), x \in B . \quad \theta$ is $C_{H}^{2}$ with $\left|\theta^{\prime}(x)\right| \leq C_{1}\|x\|\left(1+\|x\|^{2}\right)^{-1}$ and $\left\|\theta^{\prime \prime}(x)\right\|_{1} \leq C_{2}\left(1+\|x\|^{2}\right)^{-1}$, where $C_{1}$ and $C_{2}$ are two constants independent of $x$ and $\|\cdot\|_{1}$ denotes the trace class norm. Apply Ito's formula (Theorem 4.1 [14]) to the function $\theta$ and the process $X(t)$,

$$
\begin{gathered}
X(t)=x+\int_{0}^{t} A(X(s)) d W(s)+\int_{0}^{t} \sigma(X(s)) d s . \\
\theta(X(t))=\theta(x)+\int_{0}^{t}\left(A^{*}(X(s)) \theta^{\prime}(X(s)), d W(s)\right) \\
+\int_{0}^{t}\left[\left(\theta^{\prime}(X(s)), \sigma(X(s))\right)+\frac{1}{2} \operatorname{trace} A^{*}(X(s)) \theta^{\prime \prime}(X(s)) A(X(s))\right] d s .
\end{gathered}
$$

It follows easily that

$$
E(\theta(X(t))-\theta(x))^{2} \leq \text { constant }=a,
$$

or

$$
E\left[\log \frac{1+\|X(t)\|^{2}}{1+\|x\|^{2}}\right]^{2} \leq a .
$$

Now, let $f \in C_{0}$ and $g=P_{t} f$. Let $\varepsilon>0$ be given and $N$ be large enough that

$$
|f(x)|<\varepsilon / 2 \quad \text { whenever }\|x\|>N \text {. }
$$

But

$$
g(x)=E_{\{\|X(t)\|>N\}} f(X(t))+E_{[\|X(t)\| \leq N\}} f(X(t)) .
$$

Hence,

$$
|g(x)| \leq \varepsilon / 2+\|f\|_{\infty} \operatorname{prob}\{\|X(t)\| \leq N\}
$$

and

\footnotetext{
* We learn the proof from Professor K. Ito through a private conversation.
} 
prob $\{\|X(t)\| \leq N\}$

$$
\begin{aligned}
& \leq \operatorname{prob}\left\{\log \frac{1+\|X(t)\|^{2}}{1+\|x\|^{2}} \leq \log \frac{1+N^{2}}{1+\|x\|^{2}}\right\} \\
& =\operatorname{prob}\left\{\log \frac{1+\|X(t)\|^{2}}{1+\|x\|^{2}} \leq-\log \frac{1+\|x\|^{2}}{1+N^{2}}\right\}, \quad\|x\|>N, \\
& \leq \operatorname{prob}\left\{\log \frac{1+\|X(t)\|^{2}}{1+\|x\|^{2}} \mid \geq \log \frac{1+\|x\|^{2}}{1+N^{2}}\right\} \\
& \leq\left(\log \frac{1+\|x\|^{2}}{1+N^{2}}\right)^{-2} \times E\left[\log \frac{1+\|X(t)\|^{2}}{1+\|x\|^{2}}\right]^{2} \\
& \leq a\left(\log \frac{1+\|x\|^{2}}{1+N^{2}}\right)^{-2} \leq \frac{\varepsilon}{2\|f\|_{\infty}} \quad \text { if }\|x\| \text { is large. }
\end{aligned}
$$

Thus for large $\|x\|$ we have $|g(x)| \leq \varepsilon / 2+\varepsilon / 2=\varepsilon$. Therefore $g \in C_{0}$.

\section{Appendix}

It is a pleasure to thank Professor Loren Pitt for pointing out the fact that $P_{t}, t \geq 0$, being strongly continuous (Theorem 10) is not obvious. We present a proof as follows.

LEMMA A.1 (Gronwall's inequality). If $h$ is a non-negative integrable function in $[0, a], a<\infty$, satisfying

$$
h(t) \leq g(t)+\alpha \int_{0}^{t} h(s) d s,
$$

where $\alpha>0$ and $g$ is integrable in $[0, a]$. Then

$$
h(t) \leq g(t)+\alpha \int_{0}^{t} e^{\alpha(t-s)} g(s) d s
$$

Proof. We can prove inductively that

$$
\begin{aligned}
h(t) \leq & g(t)+\alpha \int_{0}^{t}\left\{\sum_{k=1}^{n-1}[\alpha(t-s)]^{k} / k !\right\} g(s) d s \\
& +\alpha \int_{0}^{t} h(s)[\alpha(t-s)]^{n} / n ! d s .
\end{aligned}
$$

The conclusion then follows from Lebesgue's dominated covergence theorem.

LEMMA A.2. $E_{x}\|X(t)-x\|^{2} \leq \operatorname{ct}\left(1+\|x\|^{2}\right)$ for all $0 \leq t \leq 1$ and all $x \in B$, where $c$ is a constant independent of $t$ and $x$. 
Proof. We use the letter $c$ to stand for any constant independent of $t$ and $x$. Let $0 \leq t \leq 1$.

$$
\begin{aligned}
X(t) & =x+\int_{0}^{t} A(X(s)) d W(s)+\int_{0}^{t} \sigma(X(s)) d s \\
& =x+C W(t)+\int_{0}^{t} K(X(s)) d W(s)+\int_{0}^{t} \sigma(X(s)) d s .
\end{aligned}
$$

Hence

$$
\begin{aligned}
\|X(t)-x\|^{2} & \leq c\left\{\|C W(t)\|^{2}+\left\|\int_{0}^{t} K(X(s)) d W(s)\right\|^{2}+\left\|\int_{0}^{t} \sigma(X(s)) d s\right\|^{2}\right\} \\
& \leq c\left\{\|C W(t)\|^{2}+\left\|\left.\int_{0}^{t} K(X(s)) d W(s)\right|^{2}+\int_{0}^{t}\right\| \sigma(X(s)) \|^{2} d s\right\} .
\end{aligned}
$$

(Recall that $\|\cdot\|$ is dominated by $|\cdot|)$.

Thus after taking expectation (36) becomes

$$
\begin{aligned}
E\|X(t)-x\|^{2} & \leq c\left\{t+\int_{0}^{t} E|K(X(s))|_{2}^{2} d s+\int_{0}^{t} E\|\sigma(X(s))\|^{2} d s\right\} \\
& \leq c\left\{t+c \int_{0}^{t} E\left(1+\|X(s)\|^{2}\right) d s+c \int_{0}^{t} E\left(1+\|X(s)\|^{2}\right) d s\right\} \\
& \leq c\left\{t+\int_{0}^{t} E\|X(s)\|^{2} d s\right\} \\
& \leq c\left\{t+c \int_{0}^{t} E\left[\|X(s)-x\|^{2}+\|x\|^{2}\right] d s\right\} \\
& \leq c\left\{t\left(1+\|x\|^{2}\right)+\int_{0}^{t} E\|X(s)-x\|^{2} d s\right\} .
\end{aligned}
$$

Hence by Lemma A.1 we have

$$
\begin{aligned}
E\|X(t)-x\|^{2} & \leq c t\left(1+\|x\|^{2}\right)+c \int_{0}^{t} e^{c(t-s)} c s\left(1+\|x\|^{2}\right) d s \\
& \leq c t\left(1+\|x\|^{2}\right)+c \int_{0}^{t} e^{c(t-s)} c t\left(1+\|x\|^{2}\right) d s \\
& =c t\left(1+\|x\|^{2}\right)\left[1+c \int_{0}^{t} e^{c(t-s)} d s\right] \\
& \leq c t\left(1+\|x\|^{2}\right) .
\end{aligned}
$$

Now let $f \in C_{0}$ be also uniformly continuous. A close examination of the proof of Theorem 10 shows that given $\varepsilon>0$ there exists $N$ independent of $t, 0 \leq t \leq 1$

$$
\left|P_{t} f(x)\right|<\varepsilon / 2 \quad \text { whenever }\|x\|>N \text {. }
$$


We may as well assume that

$$
|f(x)|<\varepsilon / 2 \quad \text { whenever }\|x\|>N .
$$

Thus we have for all $0 \leq t \leq 1$

$$
\left|P_{t} f(x)-f(x)\right|<\varepsilon \quad \text { whenever }\|x\|>N .
$$

On the other hand, let $\delta>0$ be such that

$$
\|x-y\|<\delta \text { implies }|f(x)-f(y)|<\varepsilon / 2 .
$$

Then for $\|x\| \leq N$,

$$
\begin{aligned}
\left|P_{t} f(x)-f(x)\right| \leq & E_{x}|f(X(t))-f(x)| \\
= & E_{\{\|X(t)-x\|<\delta\}}|f(X(t))-f(x)| \\
& +E_{\{\|X(t)-x\| \geq \delta\}}|f(X(t))-f(x)| \\
\leq & \varepsilon / 2+2\|f\|_{\infty} \text { prob }\{\|X(t)-x\| \geq \delta\} .
\end{aligned}
$$

But

$$
\text { prob } \begin{aligned}
\{\|X(t)-x\| \geq \delta\} & \leq \delta^{-2} E\|X(t)-x\|^{2} \\
& \leq \delta^{-2} c t\left(1+\|x\|^{2}\right) \quad \text { by Lemma A.2 } \\
& \leq \delta^{-2} c t\left(1+N^{2}\right) .
\end{aligned}
$$

Therefore we can choose $t_{0}$ small enough such that whenever $t \leq t_{0}$

$$
\left|P_{t} f(x)-f(x)\right| \leq \varepsilon \quad \text { for all }\|x\| \leq N .
$$

Clearly (37) and (38) yield that

$$
\left\|P_{t} f-f\right\|_{\infty} \leq \varepsilon \quad \text { whenever } t \leq t_{0} .
$$

This establishes the strong continuity of $P_{t}, t \geq 0$.

\section{REFERENCES}

[1] Yu. L. Dalec'kii, Differential equations with functional derivatives and stochastic equations for generalized random processes (English translation), Soviet Math. Dokl., 7 (1966), 220-223.

[2] - Infinite-dimensional elliptic operators and parabolic equations connected with them (English translation), Russian Math. Surveys, 22 (1967), 1-53.

[ 3 ] I. I. Gikhman, On the theory of differential equations of random processes (in Russian), Ukr. Matem. Zhurn., 2 (1950), 37-63.

[4] - On the theory of differential equations of random processes II (in Russian), ibid. 3 (1951), 317-339.

[ 5 ] L. Gross, Abstract Wiener spaces, Proc. 5th Berkeley Sym. Math. Stat. Prob., 2 (1965), 31-42. 
[6] - Measurable functions on Hilbert space, Trans. Amer. Math. Soc., 105 (1962), 372-390.

[ 7 ] — , Potential theory on Hilbert space, J. Func. Anal. 1 (1967), 123-181.

[8] K. Ito, On a formula concerning stochastic differentials, Nagoya Math. J., 3 (1951), 55-65.

[9] - and M. Nisio, On the convergence of sums of independent Banach space valued random variables, Osaka J. Math., 5 (1968), 35-48.

[10] D. Kannan, An operator-valued stochastic integral II, Ann. Inst. Henri Poincaré, Section B, 8 (1972), 9-32.

[11] - and A. T. Bharucha-Reid, An operator-valued stochastic integral, Proc. Japan Acad., 47 (1971), 472-476.

[12] H. Kunita, Stochastic integrals based on martingales taking values in Hilbert space, Nagoya Math. J., 38 (1970), 41-52.

[13] — and S. Watanabe, On square integrable martingales, Nagoya Math. J., 30 (1967), 209-245.

[14] H.-H. Kuo, Stochastic integrals in abstract Wiener space, Pacific J. Math., 41 (1972), 469-483.

[15] — Diffusion and Brownian motion on infinite dimensional manifolds, Trans. Amer. Math. Soc., 169 (1972), 439-459.

[16] _ On operator-valued stochastic integrals, Bulletin Amer. Math. Soc., 79 (1973), 207-210.

[17] _ and M. A. Piech, Stochastic integrals and parabolic equations in abstract Wiener space, Bulletin Amer. Math. Soc. (to appear).

[18] H. P. McKean, Stochastic integrals, Academic Press, New York-London (1969).

[19] M. A. Piech, A fundamental solution of the parabolic equation on Hilbert space, J. Func. Anal., 3 (1969), 85-114.

[20] - A fundamental solution of the parabolic equation on Hilbert space II: The semi-group property, Trans. Amer. Math. Soc., 150 (1970), 257-286.

[21] — Some regularity property of diffusion processes on abstract Wiener space, J. Func. Anal., 8 (1971), 153-172.

[22] — Diffusion semigroups on abstract Wiener space, Trans. Amer. Math. Soc., 166 (1972), 411-430.

[23] A. V. Skorokhod, Introduction to the theory of random processes (English translation), Saunders Company, Philadelphia-London-Toronto (1969).

Department of Mathematics

University of Virginia

Charlottesville, Va., U.S.A. 